\title{
On Some Peculiarities of Medical Slang
}

\author{
Marianna Ohanyan \\ Yerevan State University
}

Slang $^{1}$ is one of the vehicles through which languages change and become renewed. All languages, countries and periods of history have had slang; words with varying degrees of social acceptance and popularity. All segments of society including the most educated, cultivated speakers and writers, use some slang. In fact, this is part of the definition of slang given by Galperin (1956:108). ${ }^{2}$

It is well-known that slang fills a necessary niche in all languages, occupying a middle ground between the standard and informal words accepted by the general public and the special words and expressions known only to comparatively small social subgroups. Slang tends to originate in subcultures within a society. Occupational groups (e.g. loggers, police, medical professionals and computer specialists) are prominent originators of both jargon and slang (Partridge 1979). Slang expressions often embody attitudes and values of group members. They may thus contribute to a sense of group identity and may convey information to the listener about the speaker's background.

Although slang gained respectability in the $20^{\text {th }}$ century, it was often loudly condemned as vulgar in the past. ${ }^{3}$ To understand slang fully, one must remember that a word's use, popularity, and acceptability can change. Words can change in social level, moving in any direction. Language is dynamic, and at any given time hundreds, and perhaps thousands, of words and expressions are in the process of changing from one level to another, becoming more acceptable or less acceptable/more popular or less popular. Slang is used for many purposes, but generally it expresses a certain emotional attitude; the same term may express diametrically opposed attitudes when used by different people. The processes by which words become slang are the same as those by which other words in the language change their form or meaning or both. In addition to occupational and professional groups, there are many other types of subcultures that supply slang. These include sexual deviants, narcotic addicts, ghetto groups, institutional populations, agricultural subsocieties, political organizations, the armed forces. The professions like medicine, law etc. tend to create true neologisms often based on Greek and Latin roots, but these are not major sources for slang, though nurses and medical staff adapt some medical terminology to their slang.

The oldest written sources of western medicine are the Hippocratic writings from the $5^{\text {th }}$ and $4^{\text {th }}$ centuries B.C. which cover all aspects of medicine at that time and contain numerous medical terms. This was the beginning of the Greek era of the language of medicine, which lasted even after the Roman conquest, since the Romans, who had no similar medical tradition, imported Greek medicine. There is no recognized discipline called medical linguistics, but perhaps, as many linguists think, there ought to be one. The language of medicine offers intriguing challenges both to medical historians and to linguists (Spears 1982).

In medical practice the use of slang is widely used. Medicine is a profession already overflowing with acronyms, technical terms and Greek and Latin borrowings and doctors over the years have intended plenty of their own. Medical slang is directed at colleagues 
rather than patients. It is the one used by doctors, nurses, paramedics and other hospital and medical staff. The central aspect of medical slang is the use of facetious but impressivesounding acronyms (1), formed from the first letters of the words that make up the name of something, and invented terminology (2) to describe patients, co-workers or tricky situations. One part of medical slang is the use of technical-looking acronyms to describe patients, co-workers or situations, often facetiously, to other medical professionals. Medical slang has a growing vocabulary, yet its use in Britain remains mostly overlooked by mainstream medical literature. In other words, medical slang serves as a convenient code between medical professionals. Nevertheless, it tends to be restricted to oral use and to informal notes as, if used widely, it can lead to misunderstanding for the patient and the relatives.

Health and care professionals need good communication skills to develop positive relationships and share information with people using services. Interpersonal skills are also of great importance, the doctor should be able to communicate successfully with the patients and the relatives. Slang sometimes insults or shocks when used directly, some terms euphemize a sensitive concept. Slang is, on the one hand, thought to overcome anxieties encountered within normal medical practice (Coombs et al. 1993). These anxieties may arise as a result of clinical and diagnostic uncertainty, the difficulty in treating fellow human beings, and to distance oneself from disease and death. Gordon (1983), however, does not advocate any of these. He believes that the use of hospital slang merely facilitates interrelations among staff, thereby allowing social grouping and rapport. After all, the terms are not used in front of the patients and, if anything, suggest frustration and anger against a patient group for whom little sympathy is offered.

As mentioned before, medical slang contains lots of acronyms such as $A I D S, C T, M R$ and $P C R$. This acronyms present some difficulty; usually the initials no longer fit when the English term is translated. As a rule such discrepancies are simply ignored. AIDS, for instance, is widely accepted and has almost become a noun in its own right, though the French and Spanish SIDA and the Russian СПИД reflect the order of the equivalent words in these languages.

While analyzing medical acronyms we have noticed an important thing: some of them denote a word in English but have quite another meaning. When doctors use dance in medical slang it has nothing to do with the act of moving when dancing in real life situations. Vomit in medical slang has nothing to do with the ejecting from the mouth as the result of involuntary muscular spasms of the stomach and esophagus. There are many other slang words which have quite a different meaning if compared to their everyday usage:

Dance: the process of trying a surgical gown behind the surgeon's back.

Teeth: everything else tried

Cats: cut all to short.

Dentist: doesn't even need treatment.

Craft: can't remember a damned thing.

Vomit: victim of modern imaging technology.

Tube: totally unnecessary breast examination.

Wall: a doctor who resists admitting patients at all costs. 
Noctor: nurse that has done a 6 week course and acts like he or she is a doctor. (This one even conveys some humorous effect.)

There are lots of death-related acronyms in medical slang:

Angel lust: a death erection.

Fubar: a patient who is so sick or trauma injured that is beyond help.

$D O A$ : dead on arrival.

$J C$ : Jesus is calling.

The notion of death is implicit in the above mentioned examples. The acronyms themselves include no notion of death but the notion is clear without any effort.

Another peculiarity of medical slang is that in colour-related slang word-combinations the colour showing component is not bound to show colour at all. These words simply make one's speech more colourful and expressive.

Code yellow: urination emergency.

Blue pipe: vein as opposed to "red pipe" or artery.

Code pink: a likely homosexual.

Black cloud: a doctor who attracts difficult or prolonged cases or an unusually high number of call.

Blue Blower: patient with severe lung disease.

Pink puffer: patient breathing rapidly due to lung disease.

Red dot: physicians from India, relates to red dot on their forehead.

White lizard: the white coloured "cocktail" given for stomach problems.

Black lizard: the coloured "cocktail" which contains charcoal.

White mice: tampons.

The units given above are vivid examples of medical slang. Other shortened, brief and fancy-sounding examples (mostly hyphenated) include some slang terms related to a certain aspect of the medical specialist's job:

Pecker-checker: a urologist

Baby-catcher: an obstetrician

Knuckledragger: an orthopedist

Slasher: a surgeon

Captain kangaroo: the head of the pediatrics department

Humpty-dumpty doctor: a physiatrist or rehabilitation physician

During the whole period of medical history the basis for the relations between the doctor and the patient has always been based on confidence. The success in treatment is possible only through the combination of human confidential relationships and good scientific acquisition. A technically qualified doctor should not only treat the patient but also should have the ability to speak to his patient. Thus, medical slang being used by doc- 
tors, nurses, paramedics and other hospital and medical staff is a kind of a secret language code shared by all these people.

Notes:

1. Ernest Weekley, in his "Etymological Dictionary of Modern English" suggests that the word slang may have some relation to the verb to sling and cites two Norwegian dialect words, based upon the cognate verb slenge or slengje that appear to be its brothers (Weekly 1921:48).

2. Galperin, I.R. (1956) states that slang is words and phrases used in the sphere of colloquial speech as colloquial neologisms, and this units easily transfer into the sphere of generally used colloquial vocabulary.

3. Greenough, J.B. and Kitteridge, C.L. (1925) define slang as a peculiar kind of vagabond language, always hanging on the outskirts of legitimate speech but continually straying or forcing its way into the most respectable company.

\section{References:}

1. Coombs, R.H.; Chopra, S.; Schenk, D.R. \& Yutan, E. (1993) Medical Slang and its Functions. // Social Science and Medicine, 36.

2. Galperin, I.R. (1956) O termine sleng. // Voprosi jazikoznanija. N 6. Moscow: Progress.

3. Gordon, D. (1983) Hospital Slang for Patients: Crocks, Gomers, Gorks and others. // Language in Society, 12.

4. Greenbough, J.B. and Kittelridge, C.L. (1925) Words and Their Ways in English Speech. NY: Macmillan.

5. Partridge, E. (1979) Slang Today and Yesterday. London: Routledge and Kegan Paul.

6. Spear, R.A. (1982) Slang and Euphemism. N.Y. New American Library.

7. Weekley, E. (1921) Etymological Dictionary of Modern English. London: J. Murray.

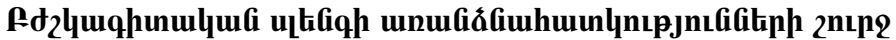

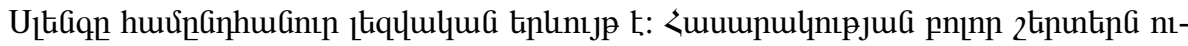

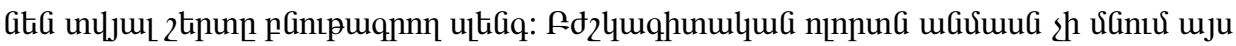

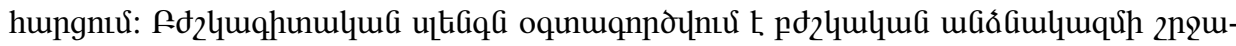

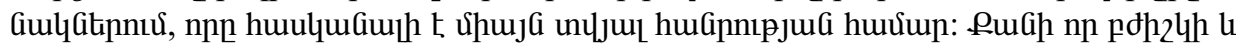

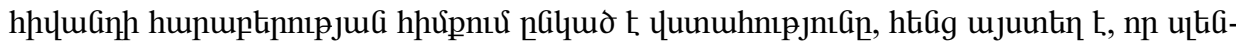

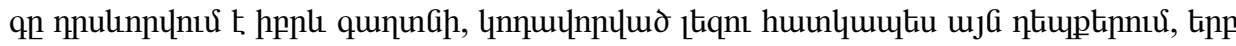

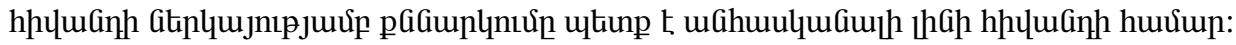

